\title{
Improved in situ performance testing of line-concentrating solar collectors: Comprehensive uncertainty analysis for the selection of measurement instrumentation
}

\author{
Annie Zirkel-Hofer ${ }^{\mathrm{a}, *}$, Stephen Perry ${ }^{\mathrm{a}}$, Sven Fahr ${ }^{\mathrm{a}}$, Korbinian Kramer ${ }^{\mathrm{a}}$, Anna Heimsath ${ }^{\mathrm{a}}$, Stephan Scholl ${ }^{\mathrm{b}}$, \\ Werner Platzer ${ }^{\mathrm{a}}$ \\ ${ }^{a}$ Fraunhofer Institute for Solar Energy Systems (ISE), Heidenhofstrasse 2, D-79110 Freiburg, Germany \\ ${ }^{\mathrm{b}}$ Insitute for Chemical and Thermal Process Engineering (ICTV), Technical University Braunschweig, Langer Kamp 7, D-38106 Braunschweig, Germany
}

\section{H I G H L I G H T S}

- Guideline for the proper selection of measurement instrumentation is presented.

- Results assure representative performance testing of concentrating collectors.

- Detailed methodology is proposed and applied to exemplary uncertainty case study.

- Complete range of different collector types and operating conditions is studied.

- One relevant gap of current testing standard for line-concentrating collectors is closed.

\section{A R T I C L E I N F O}

\section{Article history:}

Received 13 June 2016

Received in revised form 7 September 2016

Accepted 26 September 2016

Available online 14 October 2016

\section{Keywords:}

Collector testing and certification

Performance evaluation

Line-concentrating solar collectors

Uncertainty analysis

Representative test results

Selection of measurement instrumentation

\begin{abstract}
A B S T R A C T
Accurate and complete performance evaluation is playing a major role in the further development of concentrating solar collectors. To ensure dependable test results, an appropriate testing and evaluation procedure is required. Moreover, the selection and installation of suitable measurement instrumentation are essential for obtaining reliable data for the performance evaluation. The quality of the measurement instrumentation greatly influences the representativeness of the test results. Details on the measurement instrumentation recommended for the testing of low-temperature solar collectors have already been provided in the testing standard EN ISO 9806:2013. Due to the larger dimensions of concentrating collectors and thus different working temperatures and mass flow rates, these recommendations cannot be directly applied for the testing of concentrating solar collectors. A good selection of measurement instrumentation will always be a trade-off between feasibility, cost of the instrumentation and its associated uncertainties. For this reason, it is crucial for every testing and certification institution to assess the quality of measurement data during the instrumentation selection process. Until now, this aspect has been sparsely addressed in the relevant literature concerning collector testing procedures. However, uncertainty examinations have become particularly relevant for in situ testing, in which the choice of measurement instrumentation has to be adapted to the specific measurement situation on-site. In situ testing is considered to be particularly beneficial (if not even indispensable) for concentrating collectors in terms of cost effectiveness and feasibility.

With the objective of simplifying the selection of measurement instrumentation, we present an elaborate methodology and comprehensive case study concerning the uncertainty calculation of line-concentrating solar collectors. The assessment of the suitability of measurement instrumentation is conducted based on two operational reference cases. These cases adequately cover the complete range of collector types and operating conditions typically involved in the field of line-concentrating solar collectors. The analysis is designed such that the results are also transferable to other testing situations, which are not specifically studied within this publication. The presented systematic uncertainty case study thus serves as a guideline for the selection of appropriate measurement instrumentation, providing useful indications for every testing and certification entity dedicated to the planning and execution of significant and reliable collector performance testing. The associated risk decrease of performance testing is essential for the further development and economic aspects of concentrating solar technology.
\end{abstract}

(c) 2016 Elsevier Ltd. All rights reserved.

\footnotetext{
* Corresponding author.

E-mail address: annie.zirkel-hofer@ise.fraunhofer.de (A. Zirkel-Hofer).
} 


\begin{tabular}{|c|c|c|c|}
\hline \multicolumn{4}{|c|}{ Nomenclature } \\
\hline$A_{A p}$ & collector aperture area $\left(\mathrm{m}^{2}\right)$ & $u_{c}(Y)$ & combined standard uncertainty of an objective \\
\hline$c_{p}$ & specific heat capacity $\left(\mathrm{kJ} \mathrm{kg}^{-1} \mathrm{~K}^{-1}\right)$ & & function $Y$ \\
\hline$\Delta T$ & temperature difference $(\mathrm{K})$ & $\dot{V}$ & volume flow rate $\left(\mathrm{m}^{3} \mathrm{~s}^{-1}\right)$ \\
\hline$\eta$ & collector efficiency $(-)$ & $v$ & fluid velocity $\left(\mathrm{m} \mathrm{s}^{-1}\right)$ \\
\hline$G_{b}$ & direct normal irradiance $\left(\mathrm{W} \mathrm{m}^{-2}\right)$ & $X$ & measurand (random variable) \\
\hline$k$ & coverage factor & $Y$ & calculated measurand (random variable) depending on \\
\hline$k_{c}$ & normally distributed random variable representing the & & measurands $X_{i}$ \\
\hline & uncertainty of the heat capacity $c_{p}\left(T_{m}\right)$ & $y$ & estimate of measurand $Y$, result of a measurement \\
\hline$k_{\rho}$ & $\begin{array}{l}\text { normally distributed random variable representing the } \\
\text { uncertainty of the fluid density } \rho\left(T_{m}\right)\end{array}$ & COP21 & $\begin{array}{l}\text { United Nations Framework Convention on Climate } \\
\text { Change, 21st Conference of the Parties }\end{array}$ \\
\hline$\dot{m}$ & mass flow rate $\left(\mathrm{kg} \mathrm{s}^{-1}\right)$ & DNI & Direct Normal Irradiance \\
\hline$Q$ & collector output power (W) & GUM & Guide to the Expression of Uncertainty in Measurement \\
\hline$\rho$ & fluid density $\left(\mathrm{kg} \mathrm{m}^{-3}\right)$ & LFC & Linear Fresnel Collector \\
\hline$T$ & temperature $\left({ }^{\circ} \mathrm{C}\right)$ & PTC & Parabolic Trough Collector \\
\hline$T_{m}$ & mean fluid temperature $\left({ }^{\circ} \mathrm{C}\right)$ & RMS & Root Mean Square \\
\hline$U(X)$ & overall/expanded standard uncertainty of measurand $X$ & pts & points \\
\hline$u(X)$ & standard uncertainty of a measurand $X$ & & \\
\hline$U_{c}(Y)$ & $\begin{array}{l}\text { overall/expanded combined uncertainty of an objective } \\
\text { function } Y\end{array}$ & & \\
\hline
\end{tabular}

\section{Introduction}

To meet the current EU and national targets of limiting global warming and mitigating climate change according to the Paris Agreement of the UN Climate Conference (COP21), renewable energy resources will play an important role in the world's future energy mix. Due to its dispatchability in power generation and increased energy efficiency in industrial process heat, concentrating solar thermal energy is able to strongly contribute to the reduction of global $\mathrm{CO}_{2}$ emissions [1]. To legitimize investments within this sector, it is crucial to be able to reliably compare different alternatives for heat and power generation. This is only guaranteed by a viable and dependable testing of the systems in question to ensure a realistic performance prediction. Adequate testing becomes additionally relevant for the commissioning phase of newly constructed commercial systems. To this end, installed measurement instrumentation and its uncertainties represent important aspects, which are often neglected compared to other more apparent factors such as data evaluation procedures and test planning. Thus, the deliberate selection of measurement instrumentation will be specifically addressed within this publication, which has not previously been studied to this wide of an extent.

\subsection{Motivation}

A thorough testing procedure lays the foundation for a significant and comparable determination of the performance parameters of concentrating solar collectors. Representative performance parameters, such as optical efficiency and heat loss coefficients, are essential for the further development of concentrating solar collectors because they provide both indicators for meaningful comparisons between collectors and the means for performing a cost-benefit analysis of design improvements. Due to their dimensions, line-concentrating collectors can hardly be tested in laboratories and are rather tested outdoors. Larger modules, collector loops, and complete solar fields are preferentially and more appropriately tested at the production site of the manufacturer or at the final installation site of the end user, requiring in situ measurements.

Due to the absence of a laboratory facility for in situ testing, the operating conditions of the collector under test cannot be controlled because heat dissipating capacities are generally not available. This situation may require a more flexible evaluation of measurement data. Several research institutes and companies are currently investigating adequate testing and evaluation methods for line-concentrating collectors, pursuing different approaches of steady-state testing [2], quasi-dynamic testing [3] or dynamic testing $[4,5]$. A review on current testing procedures has jointly been published by experts within this working field (for an overview and details on the testing procedures, see Hofer et al. [6]), revealing a lack of standardized, flexible and applicable testing procedures for concentrating solar collectors. Therefore, national and international standardization efforts (ISO TC 180, IEC TC 117, and AENOR) are being devoted to make performance evaluation for lineconcentrating collectors more comparable and reliable, which are considered to substantially contribute to the further development and market penetration of this emerging technology.

Moreover, the lack of laboratory conditions during in situ measurements does not permit the use of standardly installed laboratory instrumentation. The sensors have to be selected according to the testing situation on-site. Differences in heat transfer media, fluid temperatures and mass flow rates should be considered for the selection of appropriate instrumentation. Additionally, specific access to the hydraulic circuit, as well as the piping material and geometry, influences the choice of suitable sensors.

The performance parameters of a collector determined from thermal measurements can only be as good as the quality of the measurement data (i.e., of the installed measurement instrumentation). The performance of a collector is recorded via measurements of, e.g., inlet and outlet temperatures of the collector, mass flow rate and ambient conditions such as direct solar irradiance and ambient temperature. The error of the test results depends on the error propagation law on the error (i.e., uncertainties) of every measurement instrument installed. Measurement instrumentation and its associated uncertainties therefore greatly influence the representativeness of the test results.

For low-temperature collectors (such as flat plate or vacuum tube collectors) tested in an indoor or outdoor laboratory (with heating and cooling capacities), recommendations for appropriate measurement instrumentation are elaborately provided in the relevant testing standard EN ISO 9806:2013 [7]. An assessment of the quality of the measurement data (i.e., of the representativeness of 
test results) is often conducted only in retrospect after installing instrumentation and collecting and evaluating the measurement data. In some cases, this uncertainty assessment is even completely neglected by referring to the recommendations of the testing standard.

Since 2013, the testing standard EN ISO 9806:2013 has also been valid for concentrating solar collectors, but its content has not been adapted to the specifics of this newly included technology, particularly when in situ testing is implemented. Solely relying on the given recommendations of the testing standard may be appropriate for the testing of low-temperature collectors, but this approach is not applicable for concentrating solar collectors. Some recommendations may not be technically feasible or may induce very high error values. In these cases, collector testing does not permit a proper characterization of the collector performance; rather, it only provides rough indications. In particular, in the context of collector testing and certification with the objective of independently determining significant and comparable performance parameters, only small error bands, e.g., approximately $1-2 \%$ of the test results, are acceptable. For this reason, it is essential for every testing and certification institution, as well as for every entity dedicated to representative and reliable performance testing, to assess the quality of the measurement data prior to actually performing in situ measurements. In fact, the associated performance uncertainties of a specific instrumentation setup need to be assessed accompanying the final selection of the measurement instrumentation to be installed. In this way, the error of the test results can be influenced and controlled beforehand to ensure representative test results in terms of evaluated collector performance parameters. In general, significant collector testing requires high-quality measurement instrumentation with high accuracy and precision. Nevertheless, a good selection of measurement instrumentation will always be a trade-off between cost of the instrumentation, feasibility of the installation and associated uncertainties.

Within this context, the present publication addresses two main aspects: first, the question of how to properly and universally calculate measurement uncertainty for concentrating solar collectors is considered. Uncertainty calculations are primarily based on the elaborate approach of the "Guide to the Expression of Uncertainty in Measurement" GUM [8], but mostly, simplifying assumptions are included regarding linearity, covariance in variables and the uncertainty associated with fluid properties. For this reason, the validity of these assumptions is checked, and a detailed methodology for a correct and comprehensive uncertainty calculation is proposed.

Second, based on the previously introduced methodology, a detailed uncertainty case study demonstrates the relevance of properly selecting measurement instrumentation for reliable performance testing. It therefore addresses the commonly raised question of why (and in which cases) standardly installed instrumentation (mostly for operation and control purposes) are not sufficiently fulfilling the requirements for representative in situ testing. The exemplary uncertainty case study compares highquality lab-testing sensors with sensors typically installed for collector maintenance and operation. Thus, the uncertainty case study serves as a guideline for the selection of adequate measurement instrumentation, providing useful indications for every testing and certification entity dedicated to the planning and execution of significant and reliable collector performance testing.

\subsection{Literature review on uncertainty analysis for solar collector testing}

Similar to the current state of available evaluation procedures for the testing of concentrating solar collectors summarized in Hofer et al. [6], the literature available in the context of measurement instrumentation and profound uncertainty analysis for solar collector testing is scarce. The testing standard EN ISO 9806:2013 [7] points to the relevance of uncertainty calculations for performance testing in general. This standard provides recommendations for the standard uncertainty of sensors to be installed, but it does not specify the overall, combined uncertainty of the test results in terms of collector output power or efficiency. To calculate combined uncertainty, it refers to the GUM [8]. This approach is pursued by several other publications: results and procedures of collector testing are reported without uncertainty examinations, solely referring to the testing standard. Fischer et al. [9] presents test results for flat plate collectors, whereas in Fischer et al. [3,10], results are introduced for small-scale parabolic trough collectors for process heat. These publications exclude specific uncertainty indications by merely citing the testing standard. Additionally, Xu et al. [11] introduces a new dynamic testing procedure for evacuated tubes but refers to the testing standard (in this case, the American equivalent ASHRAE 93-2003 [12] for details on the testing facility and measurement instrumentation. In Osório and Carvalho [13], diverse collectors (flat plate, vacuum tube and CPC collectors) are tested under transient conditions. Sallaberry et al. [14] presents the test results of a small-scale concentrating collector. In both publications, standard uncertainties of the used sensors are reported, but similar to the testing standard EN ISO 9806:2013 [7], no combined uncertainties of the test results are provided.

This reference to the test conditions according to the testing standard is even omitted for special collectors and test situations that are not covered by the testing standard. Spirkl et al. [15] at an early stage presented test results of in situ measurements but without reporting any uncertainty estimation. In Platzer et al. [16], linear Fresnel collectors are tested. Xu et al. [17] introduces a new testing procedure for parabolic trough collectors working with synthetic oil. For the testing in all these publications, no fully applicable testing standard is available and no uncertainty examination is reported.

Few publications in the context of collector testing state standard uncertainties of the measurement instrumentation and overall, combined uncertainties of the test results. Nevertheless, the explicit calculation behind it and the assumptions considered are not specified. Nayak and Amer [18] presents test results of various transient testing procedures for flat plate collectors. In Heller et al. [19], a test bench for parabolic trough collectors is introduced by providing details on the test facility and exemplary test results. Valenzuela et al. [2] presents a steady-state testing procedure for parabolic trough collectors, including uncertainty estimations for the test results but excluding (as both previously introduced publications) details on the calculation of uncertainties.

Thus far, the review of the literature in the context of uncertainty estimation for collector testing shows that many different collector testings and their results have been published. However, the reporting and detailed analysis of associated uncertainties is scarce, even though it represents a major aspect regarding the representativeness of the obtained test results. In Frank et al. [20], a small-scale parabolic trough collector is tested. Standard and combined uncertainties of the test results are reported, and details of the calculation procedure are given. Nevertheless, the collector is operated with thermal oil, and the uncertainties associated with the fluid properties of this heat transfer medium are not considered. For tests operated with water, this uncertainty source is small, whereas for other less-defined media such as thermal oil, the uncertainty is relevant to include [19].

The aforementioned aspects are considered in the work performed by Janotte et al. [21,22,5] and in the guideline for acceptance testing of parabolic troughs compiled by Kearney [23]. Within these publications, standard and combined uncertainties and details on the uncertainty calculation are reported. Moreover, 
uncertainties of the fluid properties of thermal oil are considered in the uncertainty computations. Nevertheless, the approach of including fluid property uncertainty is not fully traceable and therefore not universally applicable. Commonly used, simplified approaches concerning linearity and covariance in variables for the uncertainty calculation are pursued in both publications. To date, the validity of these simplifying assumptions has not yet been proven. Additionally, the work focuses on a specific testing situation: large-scale, high-temperature parabolic trough collectors operating with thermal oil.

\subsection{Content and structure of this publication}

With the objective of broadening the context of this work, the current publication presents a more generally applicable but still detailed uncertainty analysis for the performance evaluation of line-concentrating solar collectors. It accounts for the complete range of different collector types and operating conditions typically involved in the field of line-concentrating collectors. The presented methodology and the following results reflect both linear Fresnel (LFC) and parabolic trough (PTC) collectors working with different heat transfer media (water and thermal oil) with different operating conditions of process heat and power generation.

In Section 2, the basics of uncertainty calculation are introduced and applied to line-concentrating solar collectors. The approach pursued is based on the "Guide to the Expression of Uncertainty in Measurement" GUM [8]. Because GUM represents an abstract instruction to uncertainty calculations, adaptations to the specific measurement situation need to be applied. For this reason, the proposed methodology includes specifics for line-concentrating collectors, e.g., how to correctly cover errors in the physical properties of the heat transfer fluid. This proposed approach is closely linked to the one of Janotte [5], but the calculation is entirely deduced and therefore fully traceable. The presented methodology is universally applicable to any concentrating collector test situation, including all types of heat transfer media, such as glycol, molten salt, thermal oil and others, which is not currently available.

Moreover, GUM presents a guideline for a detailed and sophisticated uncertainty calculation. In contrast, in the literature (see, e.g., $[7,5,23])$, simplified approaches are primarily used. The validity of these simplifying assumptions has not yet been proven in the context of concentrating solar collectors. By comparing elaborate uncertainty computations (as described in GUM) to simplified calculations, the appropriateness of these simplifications is investigated in Section 3. In this way, a proper calculation and accordingly a proper assessment of data uncertainty is ensured. Uncertainty calculations for line-concentrating collectors additionally include assumptions concerning operating conditions. Therefore, Section 3 includes a study on the influence of operating conditions on the overall uncertainty values of the collector performance. The analysis shows that the error of the measurement data is strongly dependent on the operating conditions of the collector under test. This means that the measurement instrumentation installed in a large-scale power plant loop may not necessarily be suitable for a small-scale process heat collector. The results show, for the first time, that the differentiation into several operating conditions in the context of uncertainty analysis is essential.

Accordingly, the assessment of the measurement data quality and hence the assessment of the suitability of measurement instrumentation within the uncertainty case study in Section 4 is conducted based on two operational reference cases. This comprehensive approach lays the foundation for a general assessment of the sensor quality. It includes a systematic investigation of the influence of typical individual sensor instrumentation uncertainties on the overall uncertainty of the collector performance, which has not been presented in a similar way until now. The case study provides a good reference point for the decision if more precise (and hence, in most cases, more expensive) measurement instrumentation is decisively improving the quality of test results and is therefore worth the investment. The analysis is designed such that the results are also transferable to other testing situations, which are not specifically studied within this publication.

Finally, conclusions are drawn in Section 5 regarding important results and aspects of the uncertainty analysis. Indications and suggestions are provided regarding the selection of adequate measurement instrumentation for a reliable performance evaluation. In this way, an important gap in the current testing standard concerning line-concentrating solar collectors is closed.

\section{Methodology of uncertainty calculation}

The variables typically studied and assessed during performance testing are the collector output power $Q$ and the collector efficiency $\eta$. These quantities are not directly measured but are rather composed of several input quantities. Therefore, the uncertainty of the collector output power $Q$ or the collector efficiency $\eta$ respectively depend on the uncertainty of every measurand contributing to the final quantity. The objective of an uncertainty analysis is to evaluate the uncertainty associated with every single measurand and the effect that it has on the overall uncertainty of the objective function. This allows for a quality assessment of the measurement instrumentation (i.e., of the measurement data) and finally of the performance evaluation results. Uncertainty calculations are based on the approach of GUM [8], whose principle is presented in the following section.

\subsection{Basic principle of GUM}

\subsubsection{Standard uncertainty}

Given a quantity $X$ that has been measured with some degree of imprecision, the so-called standard uncertainty of $X$, denoted $u(X)$, has to be reported, which represents the standard deviation of the underlying distribution. This distribution has to be known or assumed. If $X$ represents the average of many repeated measurements (referred to as type A standard uncertainty in GUM), the distribution may be assumed to be normal. In the case that measurement imprecision is derived from manufacturer specification sheets (a type B standard uncertainty according to GUM), an underlying uniform distribution may often be assumed [8, 4.3.7].

\subsubsection{Combined uncertainty}

Given a calculated quantity of interest $Y=f\left(X_{1}, \ldots, X_{n}\right)$ that is a function of measurands $X_{1}, \ldots, X_{n}$, it is recommended to report the standard combined uncertainty $u_{c}(Y)$. This value represents the standard deviation of the distribution of $Y$ and is calculated via the Gaussian error propagation law:

$u_{c}^{2}(Y)=\sum_{i=1}^{n}\left(\frac{\partial Y}{\partial X_{i}}\right)^{2} \cdot u^{2}\left(X_{i}\right)$

Because Formula (1) represents the linear term of an appropriate Taylor expansion, the validity of this calculation is closely related to whether the objective function $Y$ is approximately linear where it is evaluated. Furthermore, it is assumed that the input measurands $X_{i}$ are uncorrelated. In the event that either of these conditions (linearity and non-covariance) are seriously violated, GUM provides further suggestions for calculating the combined uncertainty (see Section 2.3 and [8]). 


\subsubsection{Expanded/overall uncertainty}

Combined uncertainty can be translated into the language of confidence intervals. Assume that $Y$ is normally distributed with standard deviation $u_{c}(Y)$, and let $y$ be some measured instance of $Y$. Then, the interval $y \pm k \cdot u_{c}(Y)$ represents an approximate 95\% confidence interval for measurand $Y$ when we set the coverage factor $k=2$. The value $U_{c}(Y)=k \cdot u_{c}(Y)$ is called the expanded (or overall) uncertainty and is only meaningful when the coverage factor is specified. Within this publication, all expanded uncertainty figures will be reported with the coverage factor $k=2$.

\subsection{Application to line-concentrating solar collectors}

The parameters of interest in the context of performance testing are the collector output power $Q$ and the collector efficiency $\eta$. These quantities are not measured but are actually calculated with the help of several input quantities via the following formulas:

$Q=\dot{m} \cdot c_{p} \cdot \Delta T$,

and

$\eta=\frac{\dot{m} \cdot c_{p} \cdot \Delta T}{A_{A p} \cdot G_{b}}$

where

$\dot{m}=$ mass flow in $\mathrm{kg} / \mathrm{s}$,

$c_{p}=$ fluid specific heat capacity in $\mathrm{J} /(\mathrm{kg} \mathrm{K})$,

$\Delta T=$ temperature difference $T_{\text {out }}-T_{\text {in }}$ in $\mathrm{K}$,

$A_{A p}=$ collector aperture area (a constant) in $\mathrm{m}^{2}$,

$G_{b}=$ direct normal irradiance DNI in $\mathrm{W} / \mathrm{m}^{2}$.

Note that the calculated quantities have to be understood as instantaneous values of power and efficiency at exemplary, predefined conditions. Associated uncertainties of general collector power and efficiency values obtained via a regression fit of a complete set of measurement data are not studied within this publication.

\subsubsection{Standard uncertainty}

Every measurand contributing to the objective function $Q$ or $\eta$ is associated with diverse uncertainty factors. In the case of collector performance testing, the associated uncertainties of the respective measurement instrumentation are specified in their technical data sheets (a type B standard uncertainty according to GUM) or are estimated on the basis of multiplicity of measurements and observed variance (type A standard uncertainty). To determine the standard uncertainty of a measurand, every uncertainty effect is added up, irrespective of whether it is estimated or extracted from a technical data sheet. Uncertainty effects contributing to a standard uncertainty of a measurand may be the sensor uncertainty itself and additional uncertainties associated with the entire measurement chain, such as data logging, display accuracy, longterm stability, non-linearity, temperature dependencies and so forth. To calculate standard uncertainty, the probability distribution of the stated uncertainty effects must also be considered (see Appendix A and [8]).

\subsubsection{Combined uncertainty}

Directly applying the Gaussian law to Formula (2) yields the combined uncertainty

$u_{c}^{2}(Q)=\left(\frac{\partial Q}{\partial \dot{m}}\right)^{2} \cdot u^{2}(\dot{m})+\left(\frac{\partial Q}{\partial c_{p}}\right)^{2} \cdot u^{2}\left(c_{p}\right)+\left(\frac{\partial Q}{\partial \Delta T}\right)^{2} \cdot u^{2}(\Delta T)$

and applying it to (3) provides

$$
\begin{aligned}
u_{c}^{2}(\eta)= & \left(\frac{\partial \eta}{\partial \dot{m}}\right)^{2} \cdot u^{2}(\dot{m})+\left(\frac{\partial \eta}{\partial c_{p}}\right)^{2} \cdot u^{2}\left(c_{p}\right)+\left(\frac{\partial \eta}{\partial \Delta T}\right)^{2} \cdot u^{2}(\Delta T) \\
& +\left(\frac{\partial \eta}{\partial G_{b}}\right)^{2} \cdot u^{2}\left(G_{b}\right)
\end{aligned}
$$

To utilize Formulas (4) and (5), one must determine the standard uncertainties of the variables $\dot{m}, c_{p}, \Delta T$ and $G_{b}$. However, these computations depend on whether the variables are measured directly or are themselves calculated quantities as, e.g., in the case of measuring volume flow rather than mass flow (where $\dot{m}=\rho \cdot \dot{V}$ ). In the latter case, one must again apply the error propagation formula until arriving at directly measured quantities.

\subsection{Issues and particularities}

Although Formulas (4) and (5) are straightforward and convenient, they must be applied with some degree of caution. First, special attention must be paid when calculating the uncertainty of fluid properties, such as $u\left(c_{p}\right)$ and $u(\rho)$, to ensure that uncertainty arising from the use of approximate formulas is included. Second, it may be the case in certain measurement scenarios that the input quantities $\dot{m}, c_{p}$ and $\Delta T$ are correlated, depending on how these quantities are determined. Third, the objective functions $Q$ and $\eta$ are non-linear in all variables, and thus, the effect of truncating the Taylor expansion should be considered. Details concerning the consideration of these three conditions and their influence on uncertainty calculations are discussed within the following sections.

\subsubsection{Uncertainties in fluid property formulas}

The heat capacity $c_{p}\left(T_{m}\right)$ is calculated as a polynomial function of the mean fluid temperature $T_{m}$, as is the density of the heat transfer fluid $\rho\left(T_{m}\right)$, which is required when the volume flow is measured rather than directly measuring mass flow. Importantly, there is an element of uncertainty in these fluid property calculations that arises from uncertainty regarding the equations used to determine them, and this uncertainty should be factored into the calculation of $u\left(c_{p}\right)$. The magnitude of this uncertainty greatly depends on the fluid used: the evaluation of the fluid properties of water are extremely sophisticated, leading to small uncertainties (approximately $0.02 \%$ [7]), whereas for thermal oil and other heat transfer media, the uncertainty is more pronounced (approximately, e.g., 1-3\% for thermal oil [24])). To enable an uncertainty calculation that includes error in the fluid property equations, we define a quantity $c_{p}^{*}$ via the rule

$c_{p}^{*}\left(T_{m}\right)=k_{c} \cdot c_{p}\left(T_{m}\right)$,

where the factor $k_{c}$ represents a uniformly distributed random variable over the interval $1 \pm \varepsilon$. The value $\varepsilon$ represents the (relative) uncertainty of the fluid formula and is taken from documentation concerning the $c_{p}\left(T_{m}\right)$ formula used (e.g., $3 \%$ uncertainty $\rightarrow \varepsilon=0.03$ ).

This concept is sketched in Fig. 1, where the quality of the fitted function $c_{p}\left(T_{m}\right)$ in comparison to reality is expressed via the coverage region $c_{p}^{*}\left(T_{m}\right)$ depending on the random variable $k_{c}$. We always evaluate $k_{c}=1$ such that the coverage region of $c_{p}^{*}\left(T_{m}\right)$ is centered around $c_{p}\left(T_{m}\right)$. The uncertainty of the heat capacity $c_{p}^{*}$ is thus

$$
\begin{aligned}
u^{2}\left(c_{p}^{*}\right) & =\left.\left(\frac{\partial c_{p}^{*}}{\partial T_{m}}\right)^{2}\right|_{k_{c}=1} \cdot u^{2}\left(T_{m}\right)+\left.\left(\frac{\partial c_{p}^{*}}{\partial k_{c}}\right)^{2}\right|_{k_{c}=1} \cdot u^{2}\left(k_{c}\right) \\
& =\left(\frac{\partial c_{p}}{\partial T_{m}}\right)^{2} \cdot u^{2}\left(T_{m}\right)+c_{p}\left(T_{m}\right)^{2} \cdot u^{2}\left(k_{c}\right),
\end{aligned}
$$

and the value $u\left(c_{p}^{*}\right)$ is used in lieu of $u\left(c_{p}\right)$ in Formulas (4) and (5). Note that in the last line of (7), the first term is identical to 


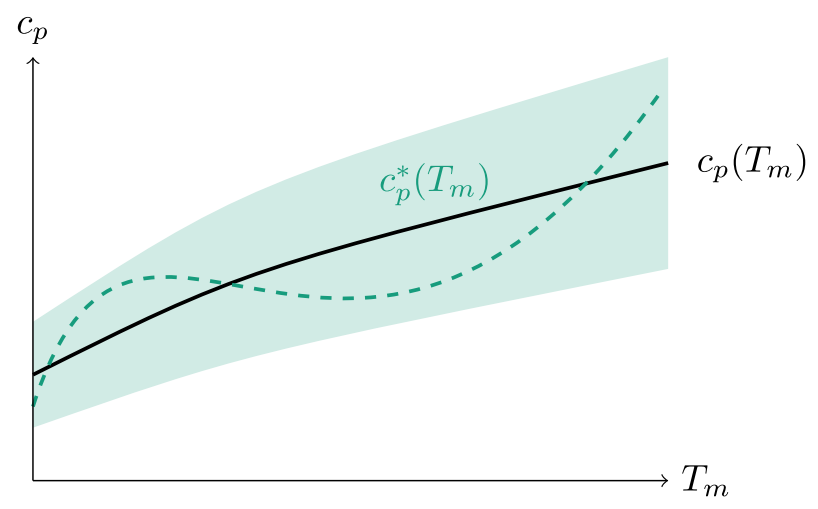

Fig. 1. Sketch of the concept of including the uncertainty of fluid property formula in uncertainty calculations (see Eq. (6)) with the true but unknown heat capacity function (dashed green line) and the approximate heat capacity function $c_{p}\left(T_{m}\right)$ (solid black line). The light green region represents possible values of $c_{p}^{*}\left(T_{m}\right)$, which is a function of the (random) variables $k_{c}$ and $T_{m}$. (For interpretation of the references to colour in this figure legend, the reader is referred to the web version of this article.)

$u^{2}\left(c_{p}\right)$; thus, the second term represents the extra variance due to formula error, where $u^{2}\left(k_{c}\right)=\varepsilon^{2} / 3$. The result of this derivation appears consistent with the computation of $u^{2}\left(c_{p}\right)$ in [5]. Within this publication, the same concept of formula uncertainty is also applied to the calculation of fluid density.

Remark. The technique of including "errors in formulas" in an uncertainty analysis must be applied with caution: once a formula has been determined, the associated uncertainty represents a type of systematic error in which the error is a function of inputs (e.g., temperature). Although this error is likely negligible when highly accurate formulas are used (e.g., the specific heat capacity of water), we consider this error in uncertainty calculations because for more inaccurate formulas (e.g., in the case of thermal oil), this error can become significant. If the operating conditions are essentially constant, this error may even cause severe bias and influence the identification results. In this case, corrections of this systematic error have to be considered.

\subsubsection{Effect of covariance}

As indicated above, the heat capacity $c_{p}\left(T_{m}\right)$ is in fact calculated as a polynomial function of the mean fluid temperature $T_{m}$. This also applies to the density calculation of the heat transfer fluid $\rho\left(T_{m}\right)$, which is required when the volume flow $\dot{V}$ is measured rather than directly measuring mass flow. Since $c_{p}\left(T_{m}\right), \dot{m}=$ $\dot{V} \cdot \rho\left(T_{m}\right)$ and $\Delta T$ are all functions of $T_{\text {in }}$ and $T_{\text {out }}$, covariance between these variables has the potential to invalidate Formulas (4) and (5).

While correlation coefficients could be considered to account for the covariance of these factors [see 8], these coefficients are impractical to determine and will likely lead to an unnecessarily poor combined uncertainty calculation. The obvious alternative is to apply the Gaussian error propagation formula with respect to truly independent input variables at the cost of more computational complexity. This means that the output quantity $Q$ (and similarly $\eta$ ) need to be expressed as a function of uncorrelated input quantities such as $T_{\text {in }}, T_{\text {out }}$ and mass flow $\dot{m}$ (or volume flow rate $\dot{V}$ or fluid velocity $v$, depending on the measurement concept). For example, the formula

$Q\left(T_{\text {in }}, T_{\text {out }}, \dot{V}\right)=\underbrace{k_{\rho} \cdot \rho\left(T_{m}\right) \cdot \dot{V}}_{\dot{m}^{*}} \cdot \underbrace{k_{c} \cdot c_{p}\left(T_{m}\right)}_{c_{p}^{*}} \cdot \Delta T$

corresponds to computing $Q$ in a measurement scenario in which $\dot{m}$ is determined via volume flow measurements and density calculations.
In this case, one must include the factors $k_{\rho}$ and $k_{c}$ to account for uncertainty in the corresponding fluid property formulas. This is necessary because the quantities $u\left(c_{p}\right)$ and $u(\rho)$ will no longer appear in the formula for combined uncertainty $u_{c}(Q)$ as $c_{p}$ and $\rho$ are treated as functions rather than variables in this case. The combined uncertainty $u_{c}(Q)$ is computed with the error propagation formula with respect to independent input variables, yielding:

$$
\begin{aligned}
u_{c}^{2}(Q)= & \left(\frac{\partial Q}{\partial k_{\rho}}\right)^{2} \cdot u^{2}\left(k_{\rho}\right)+\left(\frac{\partial Q}{\partial \dot{V}}\right)^{2} \cdot u^{2}(\dot{V})+\left(\frac{\partial Q}{\partial T_{\text {in }}}\right)^{2} \cdot u^{2}\left(T_{\text {in }}\right) \\
& +\left(\frac{\partial Q}{\partial T_{\text {out }}}\right)^{2} \cdot u^{2}\left(T_{\text {out }}\right)+\left(\frac{\partial Q}{\partial k_{c}}\right)^{2} \cdot u^{2}\left(k_{c}\right)
\end{aligned}
$$

which we evaluate with $k_{\rho}=k_{c}=1$. Note that the partial derivatives with respect to $T_{\text {in }}$ and $T_{\text {out }}$ in Formula (9) are considerably more elaborate than those in Formulas (4) and (5). For this reason, a study is presented in Section 3.2 to analyze whether such a complex calculation eliminating covariance effects is necessary or whether it is justified to neglect covariance effects between $\dot{m}, c_{p}$ and $\Delta T$ in uncertainty calculations for $Q$ and $\eta$.

\subsubsection{Effect of non-linearity}

The correctness of Formulas (4) and (5) depends on the effect of truncating higher order terms of the Taylor polynomial in the error propagation law. Since $Q$ and $\eta$ from Formulas (2) and (3) are nonlinear in all variables, the magnitude of this effect should be investigated. As the effect is likely to be more pronounced in the case of $\eta$, following the approach in GUM [8], the next most important terms are added to Formula (5) to obtain

$$
\begin{aligned}
u_{c}^{2}(\eta)= & \left(\sum_{i=1}^{4} \frac{\partial \eta}{\partial x_{i}}\right)^{2} \cdot u_{c}^{2}\left(x_{i}\right) \\
& +\sum_{i=1}^{4} \sum_{j=1}^{4}\left[\frac{1}{2}\left(\frac{\partial^{2} \eta}{\partial x_{i} \partial x_{j}}\right)^{2}+\frac{\partial \eta}{\partial x_{i}} \frac{\partial^{3} \eta}{\partial x_{i} \partial^{2} x_{j}}\right] \cdot u^{2}\left(x_{i}\right) u^{2}\left(x_{j}\right)
\end{aligned}
$$

where $x_{i}$ represents the variables $c_{p}, \dot{m}, \Delta T$ and $G_{b}$. This calculation is considerably more elaborate, and the additional uncertainty resulting from the consideration of these non-linear terms is presented in Section 3.2.

\section{Analysis of assumptions included in uncertainty calculations}

In Section 2, the details of the methodological approach pursued within the present publication are described. The calculation of individual uncertainty values of single measurement sensors and the computation of overall uncertainty values of the complete instrumentation setup serve as criteria for assessing the quality of measurement data. This allows an assessment of the quality and thereby the representativeness of the test results. The smaller the uncertainty of the collector power and efficiency, the more representative and thus dependable are the results, which is desired in a meaningful collector performance testing. However, in uncertainty calculations, several assumptions are included. To be able to calculate absolute uncertainty values, boundary conditions have to be set for which the calculation is performed. Clearly, the magnitude of the fixed boundary conditions will influence the magnitude of uncertainty. For this reason, in the case of calculating collector power and efficiency uncertainties, the influence of the collector operating conditions is studied. To this end, a meaningful assessment of measurement instrumentation is warranted. Moreover, the mathematical equations used for computing uncertainty values are based on simplifying assumptions regarding linearity and covariance. To ensure a proper calculation of uncertainty values, the validity of these assumptions is investigated in detail in the following section. 


\subsection{Influence of operating conditions}

For an uncertainty analysis of performance testing, a base case of measurement conditions has to be set for which the uncertainties of $Q$ (respectively $\eta$ ) are evaluated. A common approach is to consider typical and prevailing operating conditions of the collector under test. The range of possible operating conditions for line-concentrating solar collectors is rather large. Small-scale collectors (mostly used for process heat generation) have smaller dimensions (i.e., aperture areas) and often lower concentration ratios. This leads to smaller working temperatures and temperature differences (approximately $100-250{ }^{\circ} \mathrm{C}$ with $\Delta T$ of $10-20 \mathrm{~K}$ ), mass flow rates (approximately $0.9-2.0 \mathrm{~kg} / \mathrm{s}$ ), and collector output powers $Q$ of $70-200 \mathrm{~kW}$ [25]. In contrast, large-scale collectors are generally used for electricity generation and primarily operate at fluid temperatures of $250-500{ }^{\circ} \mathrm{C}$, temperature differences up to $100 \mathrm{~K}$ and at mass flow rates of approximately $6.5 \mathrm{~kg} / \mathrm{s}$ with collector output powers of whole loops of approximately 1-2 MW [5]. Since the uncertainty of most sensors depends on the magnitude of the measurand, the uncertainties $u_{c}(Q)$ and $u_{c}(\eta)$ are in fact functions of the operating conditions. For this reason, the influence of operating conditions on the uncertainty of the collector output power is investigated.

Fig. 2 depicts the overall, combined uncertainty $(k=2)$ of the collector output power relative to the output power $U_{c}(Q) / Q$ depending on operating conditions such as mass flow rate $\dot{m}$ and temperature difference $\Delta T$. Two different exemplary measurement instrumentation setups (consisting of temperature and mass flow sensors) are considered. Case (a) corresponds to measurement equipment based on invasive, built-in instrumentation, whereas case (b) is based on clamp-on measurement equipment, where all instrumentation and sensors do not intrude into the hydraulic fluid circuit. Details on the associated uncertainties of the implemented sensors are listed in Appendix B. In Fig. 2, the front corner of each plot represents typical operating conditions of power plant collector loops, whereas in the back corner, typical operating conditions of process heat collectors are prevailing. For case (a) in Fig. 2a, the relative overall measurement uncertainty increases only slightly from the operating conditions of power plant collector loops with values of approximately $\pm 1 \%$ to operating conditions of process heat collectors with uncertainty values of approximately $\pm 1.5-2.5 \%$. This effect is more pronounced for case (b) of clampon instrumentation, as shown in Fig. 2 b. The slope of the relative uncertainty from the solar field to process heat conditions is steeper, leading to an increase in the overall uncertainty values from $\pm 2 \%$ to a maximum of $\pm 10 \%$ for a very small-scale process heat collector. As large uncertainty values (i.e., error bands) are certainly impeding a significant and characteristic identification of performance parameters, they have to be avoided in a proper selection of measurement instrumentation. The results show that measurement instrumentation installed in a large-scale power plant loop may not necessarily be suitable for a small-scale process heat collector. Accordingly, the suitability of a set of measurement instrumentation in terms of uncertainty values strongly depends on the application and the prevailing operating conditions of the system under test. For general conclusions on the quality of measurement instrumentation and their setups, it is therefore not always sufficient to consider only one base case of operating conditions. For this reason, different operating conditions representing the entire range of lineconcentrating collectors are included in the following studies.

\subsection{Influence of covariance and non-linearity}

The uncertainty calculation not only includes assumptions regarding the operating conditions, as shown in the previous

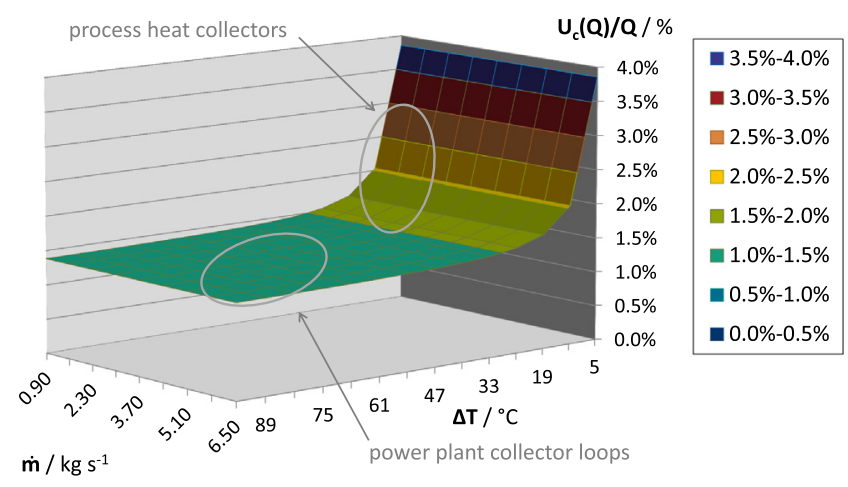

(a) Case of invasive measurement instrumentation

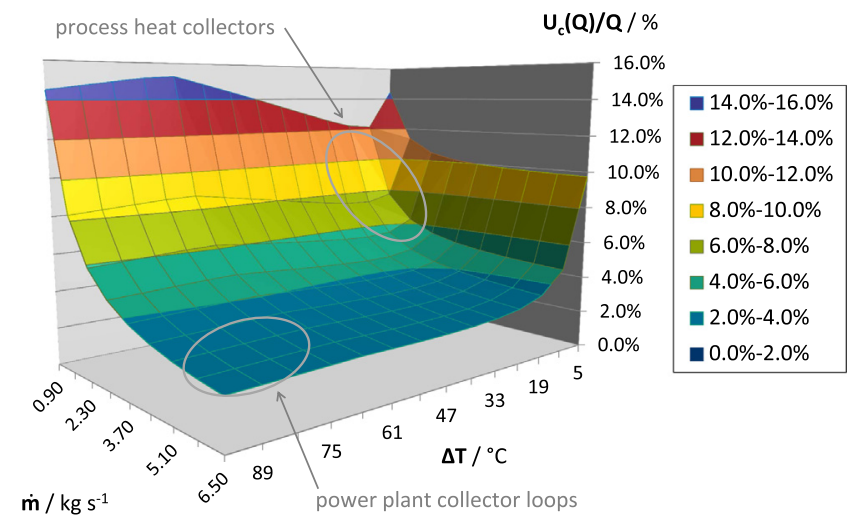

(b) Case of clamp-on measurement instrumentation

Fig. 2. Influence of operating conditions (mass flow rate $\dot{m}$ and temperature difference $\Delta T$ ) on the relative, combined measurement uncertainty of the collector output power $U_{c}(Q) / Q$ for the two measurement setups of (a) invasive and (b) clamp-on instrumentation.

section, but also methodological and computational assumptions, as described in Section 2.3. Therefore, the appropriateness of simplifying assumptions concerning the linearity of the objective function and covariance of the measurands are investigated in detail. In this way, we ensure a proper calculation and accordingly a proper assessment of data uncertainty.

\subsubsection{Non-linearity}

Although Formulas (2) and (3) for $Q$ and $\eta$ are non-linear in all variables, simplified computations of $u_{c}(Q)$ and $u_{c}(\eta)$ via the firstorder error propagation law are commonly used in the literature [see 7,5]. Therefore, calculations including higher order terms for the Taylor polynomial in the error propagation (see Formula (10)) are compared with calculations based on only first-order terms (see Formula (5)). Fig. 3 shows the differences of the uncertainty calculations for $\eta$ of both calculations. The associated sensor uncertainties are listed in Table B.3 of Appendix B.

Depending on the operating conditions for the uncertainty analysis, the differences add up to a maximum of $\pm 0.045 \%$-points of $\eta$. Uncertainty computations are conducted with respect to clamp-on measurement instrumentation because the uncertainty values of this measurement scenario are generally higher, and therefore, the deviation in the uncertainty calculation is more pronounced. The differences for a high-precision invasive measurement instrumentation would even be smaller. The mean difference amounts to only $\pm 0.003 \%$-points, indicating that the effect of non-linearity for the efficiency equation is negligible. Hence, the first-order error propagation law sufficiently approximates the uncertainty for the evaluation of $\eta$ (and therefore of $Q$ as well), which means that the use of Formulas (4) and (5) is justified. 


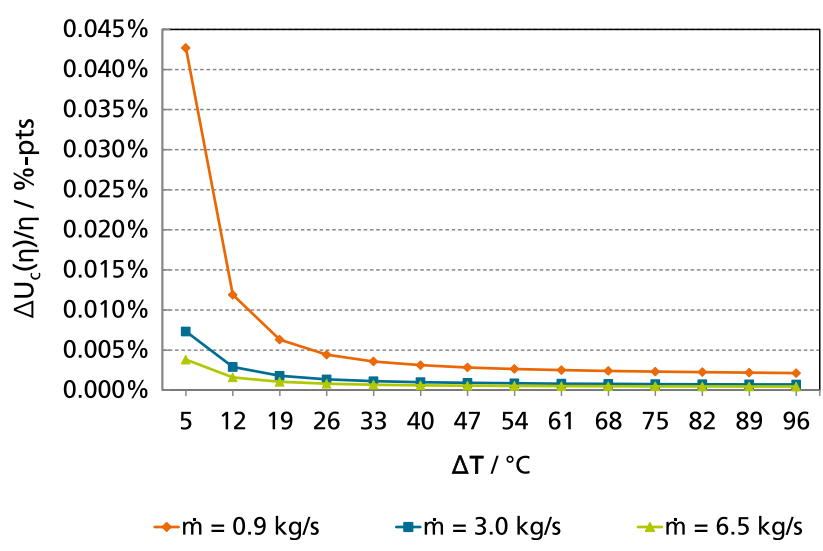

Fig. 3. Analysis of non-linearity in uncertainty calculations in terms of the relative difference of the efficiency in the uncertainty calculation $\Delta U_{c}(\eta) / \eta$ depending on the operating conditions: uncertainty calculation with higher order terms of error propagation law compared to uncertainty calculation with first-order terms only $\left(\Delta U_{c}(\eta):=U_{2 \text { nd order }}-U_{1 \text { st order }}\right)$.

\subsubsection{Covariance}

The second study was performed to evaluate the effect of covariance on the uncertainty calculation for the collector output power $Q$. Therefore, the simplified uncertainty calculation neglecting covariance effects based on Formula (4) is compared to the more precise uncertainty calculation of $u_{c}(Q)$ in Formula (9), where covariance is eliminated. The results are depicted in Fig. 4, where the difference of both uncertainty calculation approaches is shown relative to the characteristic operating conditions for lineconcentrating solar collectors. The calculations are based on the sensor uncertainties listed in Table B.3 of Appendix B associated with a clamp-on measurement scenario.

The relative deviations of the different approaches add up to a maximum of approximately $\pm 0.14 \%$-points. Again, the calculations represent an upper limit of the differences because the deviations are more distinct for clamp-on measurement instrumentation. The mean difference amounts to less than $\pm 0.09 \%$-points. In comparison to the overall measurement uncertainties of approximately $\pm 2-10 \%$, these maximum deviations are not significant and therefore justify uncertainty calculations based on the simplified approach of Formula (4).

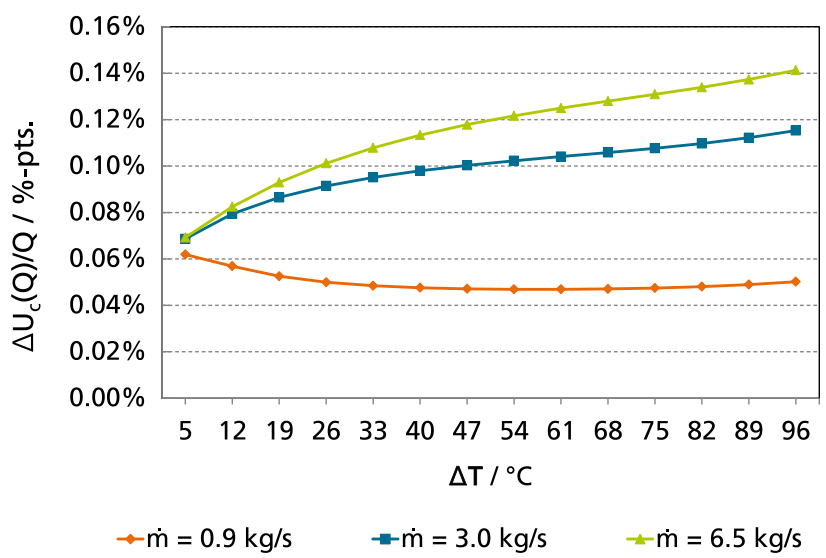

Fig. 4. Analysis of covariance in variables in uncertainty calculations in terms of relative difference of the power uncertainty calculation $\Delta U_{c}(Q) / Q$ depending on operating conditions: uncertainty calculation considering covariance effects compared to uncertainty calculation under the assumption of uncorrelated input quantities $\left(\Delta U_{c}(Q):=U_{\text {corr }}\left(Q\left(T_{\text {in }}, T_{\text {out }}, \dot{V}\right)\right)-U_{\text {uncorr }}\left(Q\left(\dot{m}, c_{p}, \Delta T\right)\right)\right)$.

\section{Uncertainty case study of typical measurement instrumentation}

The quality and thus the uncertainty of measurement instrumentation greatly influence the representativeness of test results for collector performance testing. Therefore, testing and certification entities are employing high-precision and highaccuracy sensor instrumentation for their collector testing. For indoor laboratories, standard measurement instrumentation are primarily utilized because the measurement situations of different collectors do not significantly change. For outdoor and particularly for in situ measurements, standard instrumentation cannot be directly used for every collector testing. At minimum, a re-check is necessary to determine whether the instrumentation is actually suitable for the existing measurement situation. As shown in Section 3.1, the operating conditions may severely influence the overall uncertainty values of the performance evaluation, impeding meaningful test results for certain cases. Moreover, depending on the heat transfer fluid, working temperatures and access to the site, not every standard sensor can be installed. In these cases, measurement instrumentation has to be adapted according to the specific measurement situation and the feasibility on-site. To facilitate a faster assessment and accordingly an easier selection of measurement instrumentation, exemplary measurement instrumentation typically installed for collector testing or maintenance and operation are studied. In this context, not only standard uncertainties of individual sensors are evaluated but also their impact on overall uncertainty values of collector efficiency and power. The results will indicate which sensors are dominating the overall uncertainty values and may thus be worthwhile to improve. As a starting point for the study, a base case of a standard instrumentation setup is defined, which will serve as a reference basis. For every subsequent uncertainty calculation, the sensor to be studied replaces the according sensor of the standard setup, i.e., of the base case. The different sensors evaluated represent standard measurement instrumentation typically installed in the context of performance testing or operation and control of a collector. To incorporate the effect of operating conditions, two operational reference cases are additionally specified in the following.

\subsection{Base case of measurement instrumentation and operational reference cases}

Since operating conditions may significantly influence an uncertainty evaluation, as shown in Section 3.1, for the following analysis, two different reference cases of collector sizes and their typical application are compared: a small-scale linear Fresnel process heat collector operating with pressurized water at approximately $160^{\circ} \mathrm{C}$ and a large-scale solar power plant collector loop consisting of a parabolic trough collector operating with thermal oil at temperatures of approximately $350{ }^{\circ} \mathrm{C}$. Details on the operating conditions are summarized in Table 1 . Conditions for the large-scale collector loop are analogous to those of Janotte [5]. Both cases serve as exemplary extreme scenarios, representing the entire spectrum of line-concentrating solar collector sizes and their applications in which collector performance measurements are conducted today. As a base case of the measurement instrumentation, the invasive, high-precision sensor equipment is chosen, which has already been included in the calculations and results of Fig. 2a. It involves a calibrated Pt-100 temperature sensor, a calibrated ultrasonic in-line mass flow sensor and a first class pyrheliometer for irradiance measurements.

Table 2 shows the resulting overall uncertainties of the collector output power and collector efficiency for the two operational reference cases. In both cases, the overall combined efficiency $U_{c}(\eta)$ is 
Table 1

Characteristics of the operational reference cases concerning collector size and typical operating conditions. For technical data of the used heat transfer fluids, such as density and heat capacity, see [7, p. 109] for water and [26] for thermal oil VP1.

\begin{tabular}{lrrr}
\hline Testing size & & Small-scale & Large-scale \\
\hline Collector & & LFC & PTC \\
Fluid & & WATER & VP1 \\
$T_{\text {in }}$ & ${ }^{\circ} \mathrm{C}$ & 150 & 290 \\
$T_{\text {out }}$ & ${ }^{\circ} \mathrm{C}$ & 170 & 390 \\
$\dot{m}$ & $\mathrm{~kg} / \mathrm{s}$ & 0.97 & 6.87 \\
$G_{b}$ & $\mathrm{~W} / \mathrm{m}^{2}$ & 850 & 850 \\
$Q$ & $\mathrm{~kW}$ & 84 & 1664 \\
$\eta$ & - & 0.66 & 0.65 \\
\hline
\end{tabular}

less than $\pm 1.4 \%$-points. The overall uncertainty of the collector output power with respect to the output power $U_{c}(Q) / Q$ is below $\pm 1 \%$ and $\pm 1.4 \%$ for small- and large-scale collectors, respectively. The overall uncertainties of the small-scale collector are generally smaller than for the large-scale collector loop, which at first may appear to contradict the tendency found in Fig. 2. However, the calculations from Section 3.1 reflect the fact that a single heat transfer fluid (thermal oil) was employed, whereas the present analysis concerns two cases with different fluids. Because the uncertainties associated with the calculation of the fluid properties of thermal oil are significantly larger than of water (compare with Section 2.3), uncertainties pertaining to a collector operating with water will be considerably smaller than collectors operating with thermal oil. This effect can clearly be seen by observing the shares in Table 2 that are associated with different measurands (concerning the explicit calculation of the shares, see Formula (A.5) of Appendix A). In the case of small-scale collectors, the largest shares of $U_{c}(\eta)$ belong to direct irradiance (75\%) and temperature measurement (17.8\%). For power plant collectors operating with thermal oil, DNI measurement uncertainty dominates the $U_{c}(\eta)$-budget (60\%), but specific heat uncertainty $U\left(c_{p}\right)$ accounts for $28 \%$. In the budget of $U_{c}(Q) / Q$, where DNI measurements play no role, the significance of heat capacity uncertainty increases to $70 \%$.

The stated shares are already providing indications on the relevance of the individual measurement uncertainty of specific sensor instrumentation. Although the same measurement instrumentation is used in both reference cases, particular measurands and their standard uncertainties are dominating the overall uncertainty differently. While temperature measurements are significantly influencing the overall uncertainty values of small-scale collectors (71.5\% of $\left.U_{c}(\eta)\right)$, they are less significant for large-scale collectors $\left(1.4 \%\right.$ of $\left.U_{c}(\eta)\right)$. The shares of overall uncertainty associated with different measurands allow indicative conclusions regarding which adaptations in the measurement instrumentation (with respect to higher precision of the measurand) are more effective than others and hence worth investing in. Improving a sensor that has a large share to the overall uncertainty will always be more beneficial than improving a sensor with a small share. Nevertheless, the effect of certain measurands and according instrumentation (e.g., temperature sensors) may be even higher because temperature measurements are also influencing mass flow and heat capacity uncertainties. To obtain a more comprehensive understanding of these effects, a detailed study was performed on the influence of individual sensor uncertainties on overall performance uncertainty values.

\subsection{Individual sensor uncertainty and its effect}

Calculations were performed to demonstrate the potential impact of a change in measurement instrumentation on the overall uncertainty of $Q$ and $\eta$. As previously described, the base case of measurement instrumentation is taken as a reference basis, and the effect of changing a single sensor while keeping the other sensors constant is studied. The results are depicted in Fig. 5a for small-scale and in Fig. 5b for large-scale collector performance measurements according to the operating conditions of Table 1 . Detailed sensor uncertainties can be found in Appendix B. The selected sensors represent standard measurement instrumentation typically installed for performance testing or for operation and control purposes.

For the temperature measurement, five different temperature sensors with their respective standard uncertainty $U(T)$ are considered:

- PtAC: calibrated Pt-100 sensor (reference basis)

- PtA: non-calibrated Pt-100 class A sensor

- PtB/10: non-calibrated Pt-100 sensor class 1/10-B

- Ptclamp: Pt-100 sensor mounted as a clamp-on sensor

- PtclampCr: relative calibrated Pt-100 sensor mounted as a clamp-on sensor

The results for small-scale collectors in Fig. 5a show the smallest uncertainty for the reference sensor PtAC, with an uncertainty $U(T)$ of $\pm 0.12 \mathrm{~K}$. The PtB/10-sensor may reach similar uncertainties of approximately $\pm 0.17 \mathrm{~K}$, leading to acceptable overall measurement uncertainties $U_{c}(\eta)$ of $\pm 1.4 \%$-points or $1.25 \%$ for $U_{c}(Q)$. In contrast, the uncertainties of the PtA- and Ptclamp-sensors are distinctly higher, with the Ptclamp-sensor resulting in a maximum uncertainty of $\pm 1.24 \mathrm{~K}$, corresponding to $\pm 5.59 \%$-points for $\eta$ and $\pm 8.28 \%$ for $Q$. In the studied case of an LFC process heat collector with an efficiency of $66.00 \%$, by installing a Ptclamp-sensor with an efficiency uncertainty of 5.59\%-points, the measured efficiency performance may vary between $60.41 \%$ and $71.59 \%$. Measurements with these uncertainty values only allow a rough estimation of the approximate collector efficiency. Therefore, these error bands are too high for collector certification purposes, where comparable and meaningful results are requested.

Note that the results for the uncertainty $U(T)$ represent exemplary calculations only, providing indications of the uncertainty that may be reached with this type of sensor; however, this value may not necessarily be reached in every circumstance. Sensor uncertainties highly depend on the associated uncertainties such as data logging, long-term stability and so forth (see Appendix B). Nevertheless, the study sufficiently allows conclusions to be drawn concerning the effect of $U(T)$ on $U_{c}(\eta)$ and $U_{c}(Q)$ for the different collector types. The same applies for other measurands and

Table 2

Overall, combined uncertainties of the collector output power $U_{c}(Q)$ and thermal efficiency $U_{c}(\eta)$ and its shares of measurands.

\begin{tabular}{|c|c|c|c|c|c|}
\hline \multirow{2}{*}{\multicolumn{2}{|c|}{$\begin{array}{r}\text { Testing size } \\
\text { Overall uncertainty }(2 \sigma)\end{array}$}} & \multicolumn{2}{|c|}{ Small-scale } & \multicolumn{2}{|c|}{ Large-scale } \\
\hline & & $\begin{array}{c}U_{c}(\eta) \\
\pm 1.29 \% \text {-pts }\end{array}$ & $\begin{array}{c}U_{c}(Q) / Q \\
\pm 0.98 \%\end{array}$ & $\begin{array}{c}U_{c}(\eta) \\
\pm 1.42 \% \text {-pts }\end{array}$ & $\begin{array}{c}U_{c}(Q) / Q \\
\pm 1.37 \%\end{array}$ \\
\hline \multirow{4}{*}{ Shares of } & $U(\Delta T)$ & $17.8 \%$ & $71.5 \%$ & $0.6 \%$ & $1.4 \%$ \\
\hline & $U(\dot{m})$ & $5.1 \%$ & $20.3 \%$ & $11.1 \%$ & $27.9 \%$ \\
\hline & $U\left(c_{p}\right)$ & $2.1 \%$ & $8.2 \%$ & $28.0 \%$ & $70.6 \%$ \\
\hline & $U\left(G_{b}\right)$ & $75.0 \%$ & & $60.3 \%$ & \\
\hline
\end{tabular}




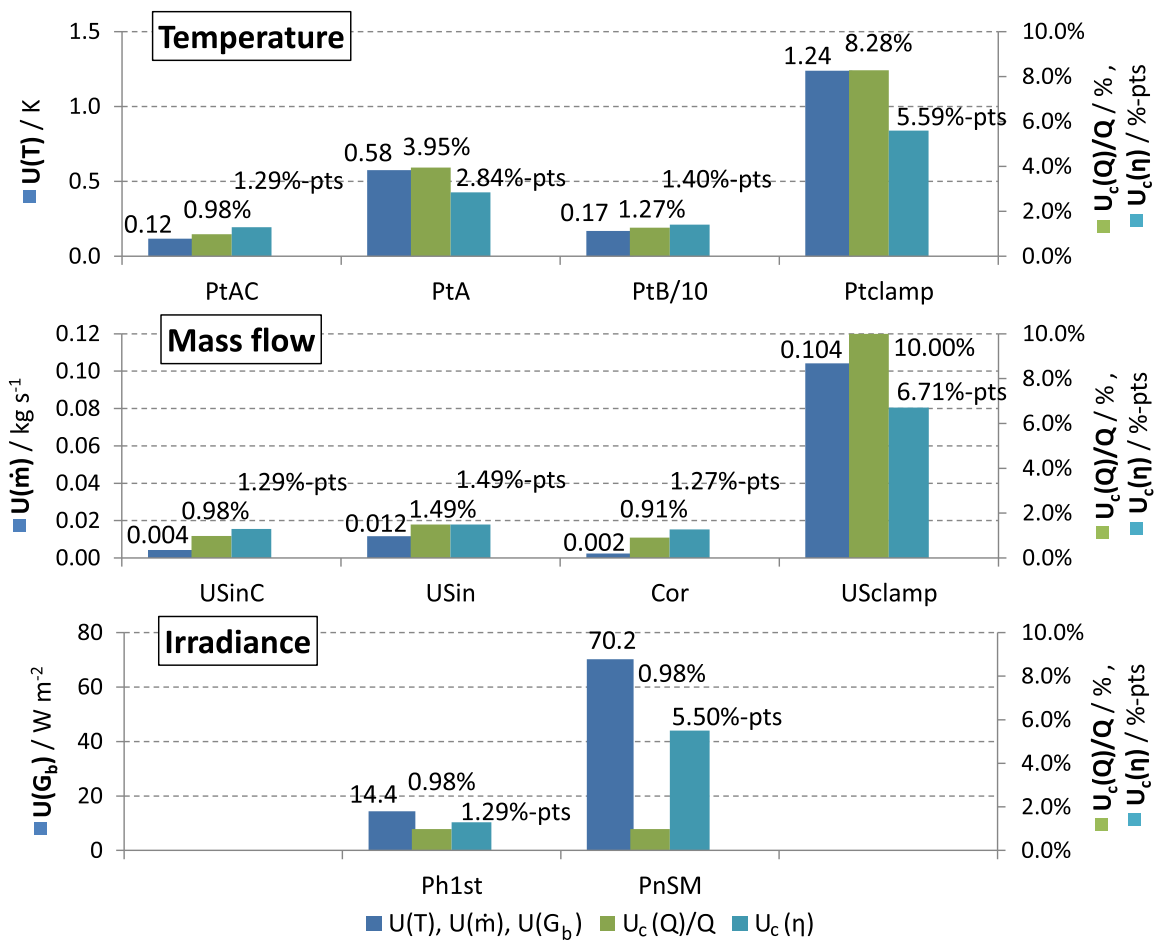

(a) Case of small-scale collector
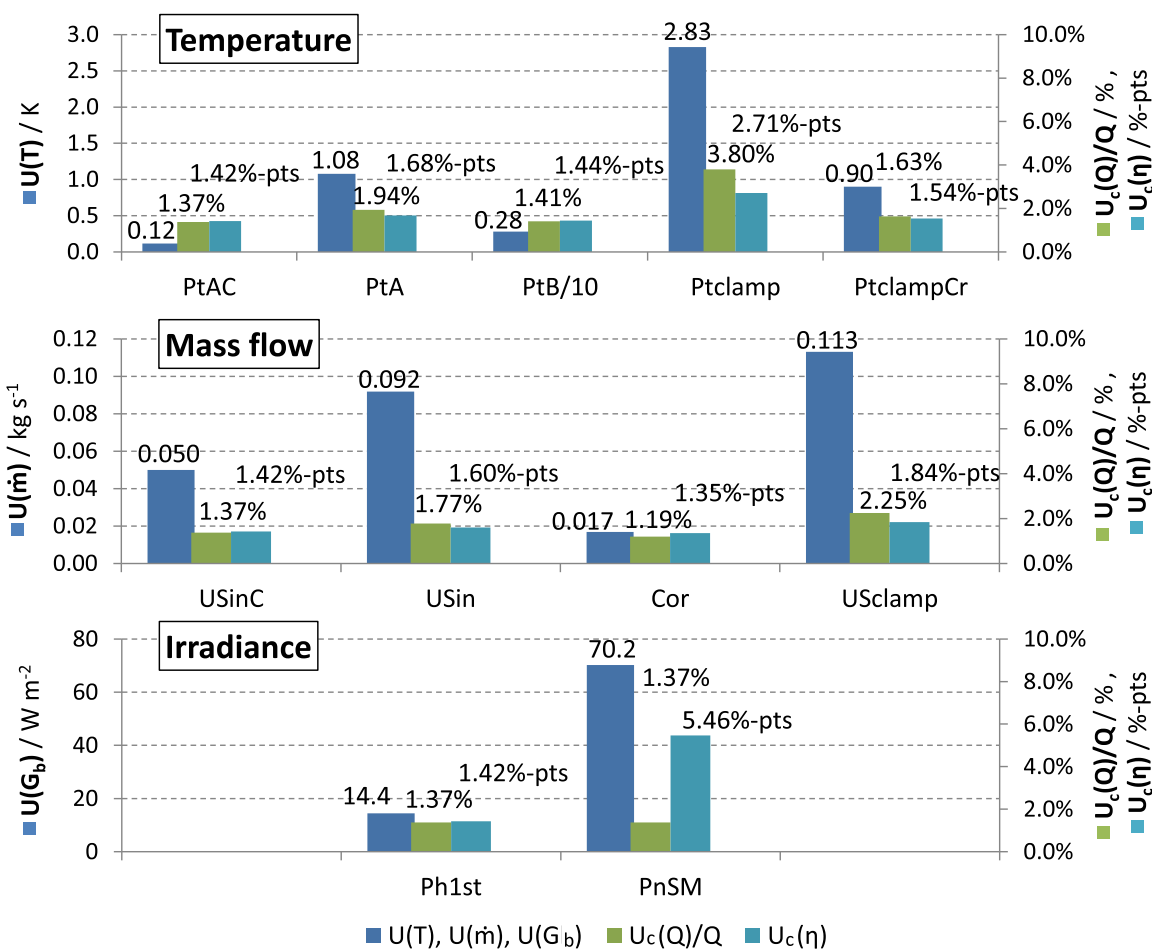

(b) Case of large-scale collector

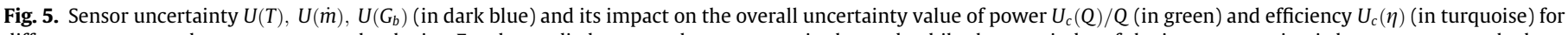

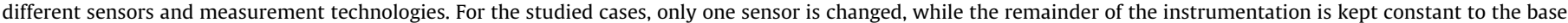

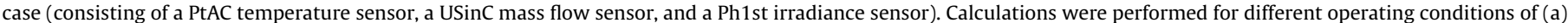
small-scale and (b) large-scale collectors. (For interpretation of the references to colour in this figure legend, the reader is referred to the web version of this article.)

their respective uncertainties, such as mass flow rate and solar irradiance.

For the large-scale system in Fig. 5b, the same tendency as for small-scale systems applies, only with a lower influence of the temperature uncertainty to overall uncertainty values: whereas temperature uncertainties of approximately $\pm 1 \mathrm{~K}$ are too high for small-scale collectors, for large-scale systems, acceptable combined uncertainties may still be reached (compare with the uncertainty of a PtA-sensor of acceptable $\pm 1.94 \%$ in $Q$ for large-scale systems but $\pm 3.95 \%$ for small-scale systems). Even significantly 
higher temperature uncertainties, as is the case with Ptclampsensors $( \pm 2.83 \mathrm{~K})$, only affect overall uncertainty values slightly, with values of $\pm 3.80 \%$ of $U_{c}(Q)$ and $\pm 2.71 \%$-points in $U_{c}(\eta)$. This tendency is consistent with the results for the uncertainty shares in Table 2, as in large-scale systems, the temperature shares are small and hardly contribute to overall uncertainties, in contrast to in small-scale systems.

Due to lower uncertainty values, for large-scale systems, clampon instrumentation represents a promising alternative to classical invasive measurement instrumentation because they do not invade into the hydraulic circuit and therefore prevail additional security risks. As today's large-scale solar field collectors have standard tube diameters and use the same thermal oil as the heat transfer fluid, clamp-on sensors may be calibrated for field applications. In the case of relative calibration, the inlet temperature sensor is calibrated with respect to the outlet temperature sensor, thus significantly decreasing the uncertainty values (see the case of PtclampCr). Small-scale process heat collectors widely differ in their absorber tube diameters, heat transfer fluids and process temperatures; therefore, this option is currently not feasible in practice for this type of collector. In combination with the option of relative calibrating the inlet and outlet temperature Ptclampsensors, an acceptable combined uncertainty $U_{c}(\eta)$ of less than $\pm 1.5 \%$-points may be reached for large-scale collector loops.

For the mass flow measurement, four different technologies with their respective expanded uncertainty $U(\dot{m})$ are considered:

\footnotetext{
- USinC: calibrated ultra-sonic in-line sensor (reference basis)

- USin: non-calibrated ultra-sonic in-line sensor

- Cor: Coriolis flow meter

- USclamp: ultra-sonic clamp-on sensor
}

In the middle section of Fig. 5a, the absolute mass flow uncertainties and the corresponding overall uncertainty values are presented. The best results can be reached with a Coriolis flow meter $( \pm 0.002 \mathrm{~kg} / \mathrm{s})$, but the uncertainty is similar to the USinCsensor $( \pm 0.004 \mathrm{~kg} / \mathrm{s})$. The corresponding overall uncertainties for both cases amount to approximately $\pm 1.3 \%$-points of $\eta$ and approximately $\pm 1 \%$ of $Q$. Not calibrating the ultra-sonic sensor (case USin) only slightly increases the overall uncertainties by $0.2 \%$-points in $\eta$ and $0.5 \%$ in $Q$. Similar to temperature measurements, the clamp-on approach (USclamp) is not applicable for small-scale process heat collectors because the uncertainty exceeds $\pm 6.7 \%$-points for the thermal efficiency ( $\pm 10 \%$ for the collector output power, respectively), precluding representative test results of performance parameters.

With respect to large-scale collectors presented in Fig. 5b, a similar tendency as in the temperature measurements is discernible: absolute mass flow uncertainty values for large-scale collectors are (to some extent significantly) higher than the ones of small-scale collectors, but the impact on overall uncertainties is smaller. This is why for large-scale collectors, USclamp-on sensors still may present a viable alternative, as the overall uncertainty of $Q$ is less than $\pm 2.25 \%$ ( $\pm 1.84 \%$-points for $\eta$ ).

For the irradiance measurement, two different sensors with their respective standard uncertainties $U\left(G_{b}\right)$ are considered:

- Ph1st: first class pyrheliometer (reference basis)

- PnSM: pyranometer with shadow mask

The results for the overall uncertainty of the collector output power $Q$ are identical for the two considered sensors because irradiance does not affect the collector output power. With a state-of-the-art first class pyrheliometer (case Ph1st), uncertainties $U\left(G_{b}\right)$ of $\pm 14.4 \mathrm{~W} / \mathrm{m}^{2}$ arise. When measuring the irradiance with an alternative pyranometer with a shadow mask, this uncertainty increases significantly to $\pm 70.2 \mathrm{~W} / \mathrm{m}^{2}$. Correspondingly, the overall uncertainty $U_{c}(\eta)$ of a small-scale collector increases from $\pm 1.29 \%$-points for a Ph1st-sensor to $\pm 5.5 \%$-points for a PnSMsensor, which may impede the proper identification of the collector efficiency parameters. Large-scale systems only show slightly lower overall uncertainty values. Note that for the DNIuncertainty calculations, only the uncertainties of the irradiance sensor have been considered, assuming a perfect maintenance (in terms of soiling of the sensor aperture) and perfect tracking of the pyrheliometer. Both factors may greatly contribute in the evaluation of collector testing if not checked and maintained on regularly basis. In the context of professional and dependable certification testing, it is assumed to be a requirement to be standardly fulfilled.

\subsection{Discussion of case study and instrumentation assessment}

The previous case study showed that high-accuracy and highprecision sensors are particularly beneficial for small-scale systems because small collectors are more sensitive to higher uncertainties of measurement instrumentation. This becomes particularly important for temperature measurements. Standardly installed temperature sensors for operation and control (as, e.g., the PtAsensor) yield high uncertainty values and accordingly impede reliable test results for small-scale systems. In the studied case of an LFC process heat collector with an efficiency of $66.0 \%$, by installing a PtA-sensor with an efficiency uncertainty of 2.8\%-points, the measured efficiency performance may vary between $63.2 \%$ and $68.8 \%$. Measurements with these uncertainty values may certainty provide valuable indications on the approximate collector efficiency. However, these error bands are still too high for collector certification purposes, where comparable results are requested. For this reason, the standardly installed instrumentation for control purposes should not be taken for representative collector testing, and the use of higher-quality sensors is essential.

In contrast, large-scale systems are less affected by high temperature uncertainties, admitting significant performance evaluation even with high temperature uncertainty values. Thus, clamp-on instrumentation can present a viable alternative to intrusive measurement instrumentation for larger systems. The same applies for mass flow measurement instrumentation. In this context, Coriolis sensors lead to the best test results because they directly measure mass flow rather than volume flow rates. However, other alternatives, such as in-line ultra-sonic sensors, may achieve similar uncertainty values and may come at lower costs and susceptibility. DNI measurement instrumentation should always be high precision because it considerably influences the identification of thermal efficiency parameters.

The recommended base case of measurement instrumentation, which is inducing acceptable overall uncertainty values, has already been implemented in practice several times in collector testing at Fraunhofer ISE. Some results of these tests have been published in Hofer et al. [4], leading to realistic and significant performance identifications. Fig. 6 shows the associated error band of the measured collector output power $Q_{\text {meas }}$ for an exemplary test day in Hofer et al. [4]. It is fit by regression to a simulated outlet power $Q_{\text {sim }}$, enabling a deduction of performance parameters of the collector under test. For this exemplary case, the regression quality in terms of the root mean square (RMS) deviation of the performance evaluation averaged to approximately $\pm 0.44 \mathrm{~kW}$. The small error band of the associated measurement instrumentation results in significant and less defective parameters being identified. Implementing other sensors that are less precise and less accurate would induce higher uncertainty values, leading to 


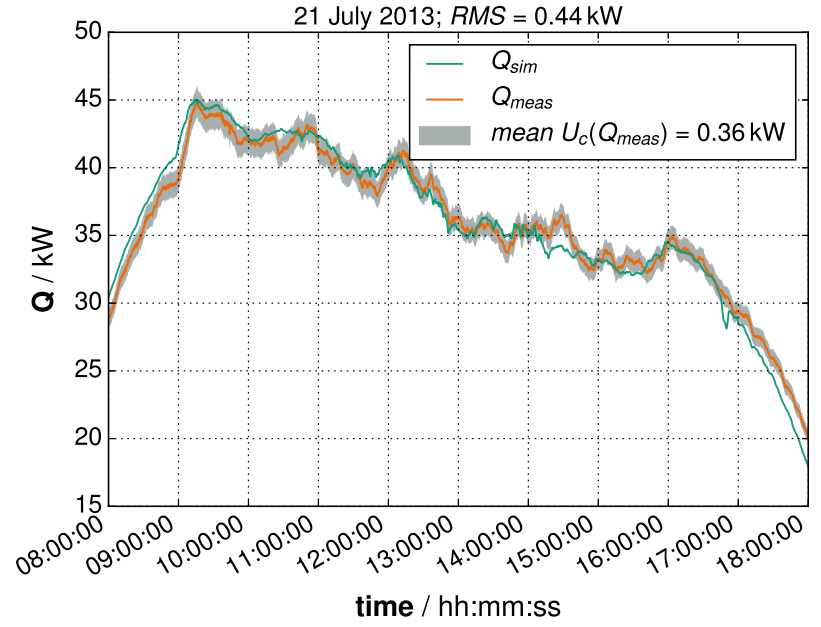

Fig. 6. Error band of measured collector output power of exemplary test collector presented in Hofer et al. [4]. Measurements were recorded with the recommended base case measurement setup of a PtAC temperature sensor, a USinC volume flow meter and a Ph1st pyrheliometer.

a poor regression quality. This is not desired in the context of professional collector testing and certification.

In general, the study confirms the relation of higher uncertainties of measurands leading to higher overall performance uncertainties and accordingly to less significant test results. Consequently, the option of improving sensor precision by, e.g., (relative) calibrating sensors should be considered, as it always diminishes overall uncertainties, particularly for smaller collectors under test. Nevertheless, sensor improvement does not always considerably enhance overall uncertainty, particularly with increasing collector powers in larger systems; thus, the extra cost and effort should be weighed against corresponding precision enhancements. This implies that for an assessment of instruments other than the ones studied within this publication, a detailed uncertainty analysis is indispensable in the context of a reliable performance evaluation. However, the denoted standard uncertainties of each sensor may provide valuable indications for other sensors as well. An alternative sensor not studied within this publication with a standard uncertainty similar to the one of a PtA-sensor and installed for the testing of a process heat collector will very likely impede representative test results, equally as the PtA-sensor. The same applies for other sensors with uncertainty values in the range of a Ptclampsensor, which may be suitable for testing of larger systems but are inappropriate for smaller collectors. Therefore, the results of Fig. 5a and $b$ represent a valuable guideline for a first classification of sensor uncertainty and eventually for a selection of adequate measurement instrumentation.

The recommended procedure for how to use the compiled methodology and its results at the beginning of a planned collector testing phase consists of the following steps:

1 Identify average values of operating conditions of the collector under test, such as mean mass flow rate, inlet and outlet temperatures and heat capacity of the heat transfer medium. Take average values of incoming irradiance depending on the potential location of the testing. From these data, deduce potential collector outlet power and efficiency:

$$
\text { test collector } \Rightarrow \bar{T}_{\text {test }}, \bar{m}_{\text {test }},{\overline{c_{p}}}_{\text {test }} \Rightarrow{\overline{Q_{\text {test }}}}, \bar{\eta}_{\text {test }}
$$

2 Compare mean values of operating conditions to the cases studied within this publication:

$$
\bar{T}_{\text {test }}, \ldots, \bar{Q}_{\text {test }} \approx / \not \approx \bar{T}_{\text {study }}, \ldots \bar{Q}_{\text {study }} ?
$$

CASE 2.1: Operating conditions are close to the small-scale or largescale test case.

- For an assessment of already installed instrumentation, compare installed sensor standard uncertainty values $U$ (sensor, e.g., T) to the indicated case study values to obtain an estimation of the induced uncertainty values on power and efficiency:

$U(T)_{\text {test }} \approx U(T)_{\text {study }} \Rightarrow U_{c}(Q)_{\text {test }} \approx U_{c}(Q)_{\text {study }}$

As previously stated, in most cases, commonly installed sensors for operation and control do not satisfy collector testing requirements.

- If induced uncertainties are too high or no instrumentation available, take the results in Fig. $5 \mathrm{a}$ and $\mathrm{b}$ and their discussion as a reference for the selection of instrumentation. For reliable collector qualification, higher-quality sensors, e.g., such as calibrated PtAC- or PtB/10 temperature sensors are recommended.

CASE 2.2: Operating conditions differ significantly from the studied test cases.

- Determine the associated sensor standard uncertainty and its effect on combined uncertainty of power and efficiency to assess instrumentation already installed:

$$
\text { sensors } \Rightarrow U(T)_{\text {test }} \underset{\text { operating conditions }}{\stackrel{\text { methodology }}{\longrightarrow}} U_{c}(Q)_{\text {test }}
$$

- If induced uncertainties are too high or no instrumentation available, take the standard uncertainty of exemplary sensors (e.g., the recommended temperature sensors PtAC or $\mathrm{PtB} / 10$ of the case study). Evaluate the associated power and efficiency uncertainty for the designated collector test according to the given operating conditions to determine whether uncertainties are in an acceptable range:

$$
U(T)_{\text {study }} \underset{\text { operating conditions }}{\stackrel{\text { methodology }}{\longrightarrow}} U_{c}(Q)_{\text {test }}
$$

3 Decide as a compromise between the cost of the instrumentation setup and minimum of associated sensor uncertainty to obtain significant and reliable collector test results.

\section{Summary and conclusion}

Investigations on testing and evaluation methods for the performance evaluation of line-concentrating collectors are diverse. Until now, the selection of adequate measurement instrumentation has remained a side issue, even though the quality of measurement instrumentation may significantly influence the representativeness of the test results. For a meaningful collector performance testing, it is not sufficient to merely state the uncertainty of the test results in retrospective after already performed measurements. In particular, in the context of comparable collector testing and certification, it is crucial to analyze associated measurement uncertainties of the test results before actually performing measurements. In fact, the calculation of measurement uncertainties needs to accompany the final selection of measurement instrumentation. Only in this way are small error bands of the results ensured. The selection of suitable measurement instrumentation becomes particularly relevant for testing on the basis of in situ measurements, where instrumentation has to be adapted to the specific measurement situation. In situ testing is considered to be particularly beneficial (if not even indispensable) for concentrating collectors in terms of cost effectiveness and feasibility.

For this reason, an elaborate methodology has been presented that focuses on diverse aspects of uncertainty calculations in a broader context of line-concentrating solar collectors. This methodology was subsequently applied for an uncertainty case 
study to assess typical measurement instrumentation, standardly available and installed for testing purposes or collector operation. To ensure a correct calculation of uncertainty values, the appropriateness of simplifying assumptions commonly applied to uncertainty calculations of collector output power and efficiency was confirmed. In particular, it was demonstrated that sophisticated computational techniques that account for non-linearity and covariance in variables in these equations do not significantly improve simplified calculations that neglect these effects.

Moreover, uncertainty results reveal the relevant influence that operating conditions have on the overall uncertainty of performance evaluations. They indicate in particular that a certain set of measurement instrumentation may be adequate for large-scale power plant collectors but may induce excessively high uncertainty values for the evaluation of small-scale process heat collectors. Thus, to draw general conclusions about the quality of specific measurement instrumentation setups, more than one fixed base case of operating conditions should be considered to account for the entire spectrum of line-concentrating solar collectors.

To facilitate a faster assessment and accordingly an easier selection of measurement instrumentation, a detailed study has been performed concerning the influence of different, individual sensor instrumentation on the overall uncertainty of the collector performance. This study is based on two operational reference cases: a small-scale process heat collector and a large-scale power plant collector loop. The results show that the overall performance uncertainties of small-scale collectors are more sensitive to uncertainties of measurement instrumentation. For small-scale collectors, temperature measurement is a major contributor to the uncertainty of the collector output power, and irradiance measurement greatly affects the uncertainty of the efficiency. To reduce the overall uncertainty for small-scale collectors, it is therefore worthwhile to improve the accuracy of these sensors, e.g., by calibrating them. The overall uncertainty values of large-scale collectors are more robust against individual sensor uncertainty. Uncertainties in power and efficiency in large-scale collectors are proportionally much smaller than in small-scale collectors. Even higher temperature uncertainties may still allow a rather decent identification of the collector performance for large-scale systems. The same applies for higher uncertainties for the mass flow rate. Conversely, uncertainty in heat capacity greatly contributes to both power and efficiency uncertainties in large-scale collectors. This implies the importance of additionally measuring this fluid property for a proper performance evaluation of a collector with less-defined heat transfer media such as thermal oil or molten salt. Irradiance measurements considerably influence efficiency uncertainty values in both small- and large-scale systems, requiring high-quality irradiance instrumentation.

In general, the study confirms the relation of higher uncertainties of measurands leading to higher overall performance uncertainties and accordingly to less significant test results. Nevertheless, the extra cost and effort have to be carefully weighed against the associated precision enhancement. For this reason, this comprehensive study provides valuable indications for future in situ collector testing with respect to the type of instrumentation advisable to select given certain boundary conditions. It furthermore shows how to proceed when selecting new measurement instrumentation for in situ testing: merely using standard sensors designed for operation and control or solely using the same instrumentation of a different collector testing does not ensure significant test results. A successful testing requires a rechecking of collector performance uncertainties and shows the indispensability of detailed uncertainty analysis in the context of reliable performance evaluations. On this account, the general methodology of uncertainty calculation for line-concentrating collectors has been introduced in detail. However, by including two operational reference cases, the present study already provides helpful orientation for the complete spectrum of line-concentrating solar collectors: a sensor with a similar standard uncertainty as a sensor studied within this publication will very likely have similar influence on the test results. In the case of a measurement situation being very different from the exemplary cases studied within this publication, the thoroughly introduced methodology can still be applied. The analysis therefore provides a good reference point for the decision if more precise (and hence, in most cases, more expensive) measurement instrumentation is decisively improving the quality of test results and is hence worth the investment. Overall, the present publication may contribute greatly to the improvement of performance testing, as the risk associated with determining collector performance can be decreased notably with an increase in the reliability of performance testing procedures. In this way, a guideline has been generated addressing specific aspects of a proper selection of convenient measurement instrumentation, which will be of particular use for every testing and certification entity dedicated to the planning and execution of significant and reliable collector performance testing.

\section{Acknowledgments}

The authors would like to thank the German Federal Ministry of Economic Affairs and Energy BMWi for the financial support in the project StaMeP, 16UM0095. This work was also partly funded by the project FRESH-NRG of the 7th Framework Programme of the European Union under Grant Agreement No. 308792.

Moreover, the authors would like to thank the Deutsche Bundesstiftung Umwelt DBU for the financial support in the context of their PhD Scholarship Programme.

\section{Appendix A. Specifics concerning uncertainty calculations}

\section{A.1. Probability distributions}

To calculate the standard uncertainties associated with a random variable $X$, probability distributions of diverse effects influencing the standard uncertainty have to be considered, as follows:

- uniform probability distribution:

$$
u^{2}(X)=\frac{u_{e f f e c t}^{2}}{3}
$$

- normal 68\%-probability distribution $(1 \sigma)$ :

$$
u^{2}(X)=u_{e f f e c t}^{2}
$$

- normal 95\%-probability distribution $(2 \sigma)$ :

$$
u^{2}(X)=\left(\frac{u_{e f f e c t}}{2}\right)^{2}
$$

Example. The standard uncertainty of a temperature sensor with $0.15^{\circ} \mathrm{C}$ of uncertainty of the reference sensor (normal distribution $2 \sigma$ ) and $0.0996^{\circ} \mathrm{C}$ of uncertainty of the data logger system (uniform distribution) is calculated as follows:

$u^{2}(T)=\left(\frac{0.15}{2}\right)^{2}+\frac{0.0996^{2}}{3}=0.078^{\circ} \mathrm{C}$

\section{A.2. Shares of uncertainty values}

The uncertainty budget for an objective quantity $Y$ consists of calculating for each $X_{i}$ its share according to: 
share of $U\left(X_{i}\right)=u_{\%}\left(X_{i}\right)=\frac{\left(\frac{\partial Y}{\partial X_{i}}\right)^{2} \cdot u^{2}\left(X_{i}\right)}{u_{c}^{2}(Y)} \cdot 100 \%$

Example. The tested collector runs with water ( $c_{p}$ of $4.33 \mathrm{~kJ} /$ $(\mathrm{kg} \mathrm{K})$ ) at a mean temperature of $160{ }^{\circ} \mathrm{C}$, a temperature difference of $20 \mathrm{~K}$ and a mass flow rate of $0.97 \mathrm{~kg} / \mathrm{s}$, leading to a power value of $84 \mathrm{~kW}$. The overall, combined measurement uncertainty of the test collector $U_{c}(Q)$ is $0.82 \mathrm{~kW}$. The mass flow sensor has a standard uncertainty of $0.004 \mathrm{~kg} / \mathrm{s}$. The share of this sensor is calculated as follows:

$\begin{aligned} u_{\%}(\dot{m}) & =\frac{\left(\frac{\partial Q}{\partial \dot{m}}\right)^{2} \cdot u^{2}(\dot{m})}{u_{c}^{2}(Q)} \cdot 100 \%=\frac{(20 \cdot 4.33)^{2} \cdot 0.004^{2}}{0.82^{2}} \cdot 100 \% \\ & =17.8 \%\end{aligned}$

Remark. The resulting percentage does not match the value presented in Table 2 due to rounding errors.

\section{Appendix B. Sensor standard uncertainty}

Uncertainty calculations within this publication include associated uncertainty effects for the particular measurands according to Tables B.4, B.5 and B.6. Table B.3 summarizes the considered measurement instrumentation for the results depicted in the corresponding figures. In the case of relative-calibrated temperature sensors, the uncertainties do not depend on single temperature measurements $T_{\text {in }}$ and $T_{\text {out }}$ but rather on the (relative) temperature difference between both. For details on the concept of relative calibration of clamp-on measurement instrumentation, see Janotte [5, pp. 154]. The associated uncertainties considered for PtclampCr-sensors are taken from that publication with a value of $u(\Delta T)=0.45 \mathrm{~K}$ assuming a temperature difference of $100 \mathrm{~K}$.

Table B.3

Considered measurement instrumentation on which results are based and presented in the stated figures.

\begin{tabular}{lcccc}
\hline & Fig. 2a & Fig. 2b & Fig. 3 & Fig. 4 \\
\hline $\mathrm{T}$ & PtAC & PtclampCr & Ptclamp & Ptclamp \\
$\dot{m}$ & USinC & USclamp & USclamp & USclamp \\
fluid & VP1 & VP1 & VP1 & VP1 \\
\hline
\end{tabular}

Table B.4

Considered uncertainty effects for the calculation of standard uncertainty for the different cases of irradiance measurement sensors.

\begin{tabular}{lcll}
\hline Uncertainty factors & Value / W & Distr. \\
\cline { 1 - 2 } Type: Ph1st & & \\
\hline Calibration & Reference sensor & $0.38 \%$ & Normal $(2 \sigma)$ \\
& Max. deviation & $1.40 \%$ & Normal $(2 \sigma)$ \\
Non-stability & $0.50 \%$ & Uniform \\
Non-linearity & $0.20 \%$ & Uniform \\
Temp. dependency & $0.50 \%$ & Uniform \\
Zero-offset & $1 \mathrm{~W}$ & Uniform \\
Data logger & $0.15 \%$ & Uniform \\
\hline Type: PnSM & & \\
\hline Sensor & $8.00 \%$ & Normal $(2 \sigma)$ \\
Non-stability & $1.00 \%$ & Uniform \\
Non-linearity & $1.00 \%$ & Uniform \\
Temp. dependency & $1.00 \%$ & Uniform \\
Zero-offset & $3 \mathrm{~W}$ & Uniform \\
Data logger & $0.15 \%$ & Uniform \\
\hline
\end{tabular}

Table B.5

Considered uncertainty effects for the calculation of standard uncertainty for the different cases of temperature measurement sensors.

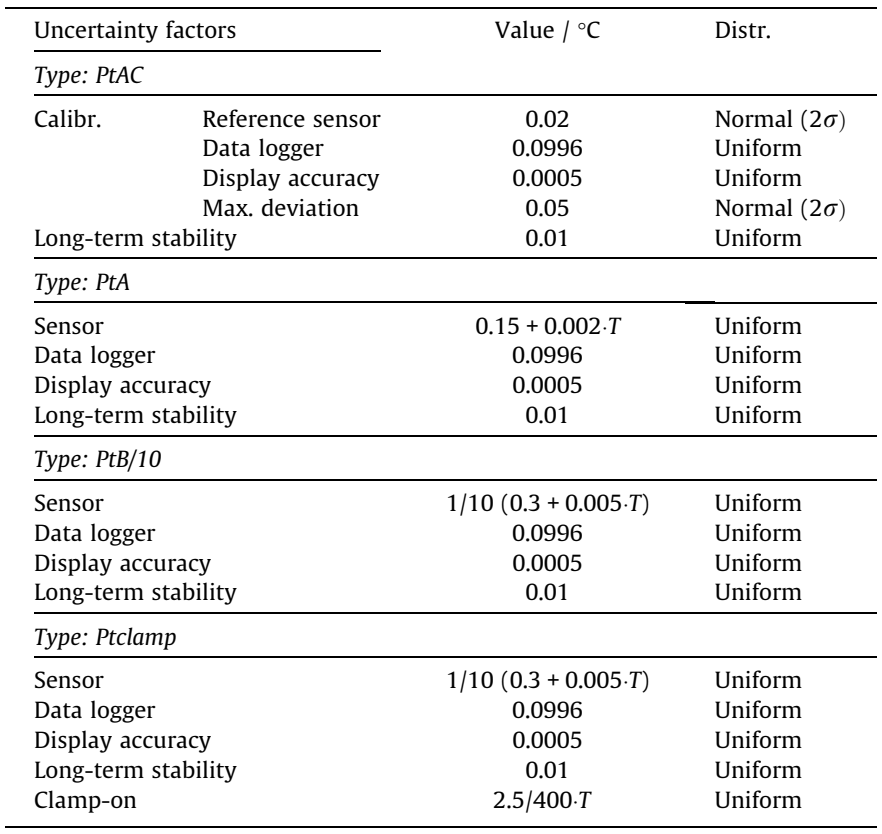

Table B.6

Considered uncertainty effects for the calculation of standard uncertainty for the different cases of mass flow measurement.

\begin{tabular}{lcl}
\hline \multicolumn{1}{l}{$\begin{array}{l}\text { Uncertainty factors } \\
\text { Type: USinC }\end{array}$} & Value $/ \mathrm{kg} / \mathrm{s}$ & Distr. \\
\hline Calibration $\quad \begin{array}{l}\text { Reference sensor } \\
\text { Max. deviation }\end{array}$ & $0.02 \%$ & Normal $(2 \sigma)$ \\
Long-term stability & $0.27 \%$ & Normal $(2 \sigma)$ \\
Data logger & $0.30 \%$ & Uniform \\
\hline Type: USin & $0.10 \%$ & Uniform \\
\hline Sensor & & \\
Long-term stability & $1.00 \%$ & Normal $(2 \sigma)$ \\
Data logger & $0.30 \%$ & Uniform \\
\hline Type: Cor & $0.10 \%$ & Uniform \\
\hline Sensor & & \\
Long-term stability & $0.15 \%$ & Normal $(2 \sigma)$ \\
Data logger & $0.15 \%$ & Uniform \\
\hline Type: USclamp & $0.10 \%$ & Uniform \\
\hline Sensor & & \\
Resol. accuracy $>250{ }^{\circ} \mathrm{C}$ & $1.2 \%$ & Uniform \\
Resol. accuracy $<250{ }^{\circ} \mathrm{C}$ & $0.01 \mathrm{~kg} / \mathrm{s}$ & Uniform \\
Long-term stability & $0.005 \mathrm{~kg} / \mathrm{s}$ & Uniform \\
Data logger & $0.15 \%$ & Uniform \\
\hline
\end{tabular}

\section{References}

[1] Teske S, Leung J. Solar thermal electricity: Global outlook 2016. Jointly prepared by European Solar Thermal Electricity Association (ESTELA), Greenpeace International and SolarPACES.

[2] Valenzuela L, López-Martín R, Zarza E. Optical and thermal performance of large-size parabolic-trough solar collectors from outdoor experiments: a test method and a case study. Solar Energy 2014(70):456-64. ISSN 0038092X.

[3] Fischer S, Lüpfert E, Müller-Steinhagen H. Efficiency testing of parabolic trough collectors using the quasi-dynamic test procedure according to the European Standard EN 12975. In: SolarPACES 2006, 13th Solar Power And Chemical Energy Systems International Symposium. SolarPACES; 2006. ISBN 8-47834519-1.

[4] Hofer A, Büchner D, Heimsath A, Fahr S, Kramer K, Platzer W, et al. Comparison of two different (quasi-) dynamic testing methods for the performance evaluation of a linear fresnel process heat collector. Energy Procedia 2015 (69):84-95. ISSN 18766102. 
[5] Janotte N. Requirements for representative acceptance tests for the prediction of the annual yield of parabolic trough solar fields. Shaker, Aachen: Berichte aus der Energietechnik; 2012. ISBN 978-3-8440-1565-2.

[6] Hofer A, Valenzuela L, Janotte N, Burgaleta J, Arraiza J, Montecchi M, et al. State of the art of performance evaluation methods for concentrating solar collectors. AIP Conf Proc 2016(1734):020010-1-0-7.

[7] ISO 9806:2013. Solar energy - solar thermal collectors - test methods; 2013. ISBN 0580790037.

[8] JCGM. Joint committee for guides in metrology: evaluation of measurement data - Guide to the expression of uncertainty in measurement (GUM); JCGM $100 ; 2008$.

9] Fischer S, Heidemann W, Müller-Steinhagen H, Perers B, Bergquist P, Hellstrom B. Collector test method under quasi-dynamic conditions according to the European Standard EN 12975-2. Solar Energy 2004;76(1-3):117-23. ISSN 0038-092X.

[10] Fischer S, Helminger F, Serrats E, Kramer K, Lampe C, Kovacs P. Topic report for WP2 Solar thermal collectors - experience from tests on concentrating and tracking collectors. Quality Assurance in Solar Heating and Cooling Technology QuAiST.

[11] Xu L, Wang ZF, Yuan GF, Li X, Ruan Y. A new dynamic test method for thermal performance of all-glass evacuated solar air collectors. Solar Energy 2012;86 (5):1222-31. ISSN 0038-092X.

[12] ASHRAE 93-2003. Methods of Testing to Determine the Thermal Performance of Solar Collectors; 2003.

[13] Osório T, Carvalho M. Testing of solar thermal collectors under transient conditions. Solar Energy 2014(104):71-81.

[14] Sallaberry F, Pujol-Nadal R, Martínez-Moll V, Torres J. Optical and thermal characterization procedure for a variable geometry concentrator: a standard approach. Renew Energy 2014(68):842-52.

[15] Spirkl W, Muschaweck J, Kronthaler P, Scholkopf W, Spehr J. In situ characterization of solar flat plate collectors under intermittent operation. Solar Energy 1997;61(3):147-52. ISSN 0038-092X.
[16] Platzer W, Heimsath A, Hülsey H. Parameter identification technique for the determination of optical efficiency of concentrating collectors. In: SolarPACES 2009, 15th Solar Power And Chemical Energy Systems International Symposium.

[17] Xu L, Wang ZF, Li X, Yuan GF, Sun FH, Lei DQ. Dynamic test model for the transient thermal performance of parabolic trough solar collectors. Solar Energy 2013;95:65-78. ISSN 0038-092X.

[18] Nayak JK, Amer EH. Experimental and theoretical evaluation of dynamic tes procedures for solar flat-plate collectors. Solar Energy 2000;69(5):377-401. ISSN 0038-092X.

[19] Heller P, Meyer-Grünefeldt M, Ebert M, Janotte N, Nouri B, Pottler K, et al KONTAS - a rotary test bench for standardized qualification of parabolic trough components. In: SolarPACES 2011, 17th Solar Power And Chemical Energy Systems International Symposium.

[20] Frank E, Marty H, Hangartner L, Minder S. Evaluation of measurements on parabolic trough collector fields for process heat integration in swiss dairies. Energy Procedia 2014(57):2743-51.

[21] Janotte N, Lüpfert E, Pitz-Paal R, Pottler K, Eck M, Zarza E, et al. Influence of measurement equipment on the uncertainty of performance data from test loops for concentrating solar collectors. In: SolarPACES 2008, 14th Solar Power And Chemical Energy Systems International Symposium. SolarPACES; 2008.

[22] Janotte N, Lüpfert E, Pitz-Paal R. Acceptance testing and advanced evaluation strategies for commercial parabolic trough solar fields. In: SolarPACES 2012, 18th Solar Power and Chemical Energy Systems International Symposium.

[23] Kearney D. Utility-scale parabolic trough solar systems: performance acceptance test guidelines, April 2009 - December 2010. National Renewable Energy Laboratory (NREL) (NREL/SR-5500-48895).

[24] Gomez J, Greg C, Mehos M. Heat capacity uncertainty calculation for the eutectic mixture of biphenyl/diphenyl ether used as heat transfer fluid; 2012

[25] Fernández-García A, Zarza E, Valenzuela L, Pérez M. Parabolic-trough sola collectors and their applications. Renew. Sust. Energy Rev. 2010 (14):1695-721.

[26] Solutia. Therminol VP-1, Heat Transfer Fluids by Solutia - Technical Bulletin. 